\title{
Relationships between Metabolic Comorbidities and Occurrence, Severity, and Outcomes in Patients with Acute Pancreatitis: A Narrative Review
}

\author{
Xu Li $\mathbb{D}^{D}$, Xiaolin Guo $\mathbb{D}^{D}$, Huifan Ji $(\mathbb{D}$, Junqi Niu $(\mathbb{D}$, and Pujun Gao \\ Department of Hepatology, The First Hospital of Jilin University, Changchun, China \\ Correspondence should be addressed to Junqi Niu; junqi_niu@163.com and Pujun Gao; gpj0411@163.com
}

Received 19 June 2019; Accepted 7 August 2019; Published 7 October 2019

Academic Editor: Roberto Cangemi

Copyright (c) $2019 \mathrm{Xu} \mathrm{Li}$ et al. This is an open access article distributed under the Creative Commons Attribution License, which permits unrestricted use, distribution, and reproduction in any medium, provided the original work is properly cited.

\begin{abstract}
Background. The population of patients with acute pancreatitis treated by the staff at our department of gastroenterology includes those with mild and self-limited disease ranging to those with severe and fatal disease. Early diagnosis and accurate prediction of the severity and outcome of this disease, which is commonly seen by our department, is important for a successful outcome. Metabolic comorbidities (e.g., diabetes mellitus, fatty liver, obesity, and metabolic syndrome) are relevant to the severity and progression of many diseases. The objective of this review was to examine clinical relationships between metabolic comorbidities and occurrence, severity, and outcome of acute pancreatitis.
\end{abstract}

\section{Introduction}

Ten to twenty percent of patients with acute pancreatitis (AP) experience failure of multiple organs [1]; there is a high mortality rate in this group of patients. The trajectory of this inflammatory disease of the pancreas depends on severity. The 2012 revised Atlanta classification of pancreatitis divides the disease into the categories of mild, moderate, or severe. Severe AP has a significant risk of mortality (up to $40 \%$ ), with the greatest mortality risk in patients with infected pancreatic necrosis [2]. Successful management of AP is challenged by the limited treatment options and the high mortality rates. Therefore, potential modifiable risk factors are still being investigated. The most common causes of AP are biliary obstruction (up to $60 \%$ of patients with AP) [3], excessive alcohol consumption (30\%) [3-5], hyperlipidemia, and autoimmune disease [6].

The values for the prevalence of chronic metabolic comorbidities such as fatty liver (FL), diabetes mellitus (DM), and metabolic syndrome have increased worldwide. Interest in the roles of comorbidities in patients with acute and critical illness has developed over the past two decades [7-10]. In 2008, 35\% of adults worldwide were overweight and $11 \%$ were obese [7]. Since 1990, the prevalence of DM has doubled worldwide; 285 million people had a diagnosis of DM in 2010 and up to $90 \%$ of these had type $2 \mathrm{DM}$ in the past three decades $[8,11,12]$. Clinical understanding of metabolic syndrome has changed since it was first defined by Reaven [13] in 1988. It is a combination of factors that includes DM, hyperglycemia, dyslipidemia, arterial hypertension, and abdominal obesity [14].

Comorbidities have long been recognized as important determinants of severity and outcome. They are included in many classifications of disease severity (e.g., the acute physiology and chronic health evaluation (APACHE) II Score) [15]. Metabolic syndrome is associated with various diseases. However, the relationships between metabolic syndrome criteria and pancreatic diseases are not well-understood [16]. Only a limited number of articles examine the relationships between metabolic comorbidities and pancreatitis (Table 1).

The aim of this review was to examine the effects of FL, $\mathrm{DM}$, and metabolic syndrome on the development, severity, and outcomes of AP.

\section{Effects of Metabolic Syndrome}

Metabolic syndrome is defined by the presence of a cluster of conditions that includes hyperglycemia, dyslipidemia, 
hypertension, and central obesity [9]. Investigation of the relationships between metabolic syndrome and the course of AP has been limited. One prospective cohort study of 140 patients in Saudi Arabia with a first attack of AP used the international diabetes federation definition of AP during investigation of the relationship between AP and metabolic syndrome [16]. They found a high prevalence (62.8\%) of metabolic syndrome among patients with AP and a high correlation between metabolic syndrome and biliary pancreatitis in men and women [17]. However, they found that metabolic syndrome does not significantly affect the severity of AP [16]. Some of the individual elements of metabolic syndrome are associated with an increased risk and more severe course of AP. In contrast to the result reported by Sawalhi et al. [16], Mikolasevic et al. found that compared with populations of patients without metabolic syndrome, populations with metabolic syndrome have significantly greater values for incidence of moderately severe and severe AP [18]. Research on the effects of metabolic syndrome on AP outcomes using large, multi-center studies is needed.

2.1. Obesity. Taken together, study results indicate that the presence of obesity has negative effects on the trajectory of AP $[16,19-24]$. Patients with obesity are at a greater risk for fatty infiltration of the pancreas. Results of clinical investigations indicate that obesity increases AP severity. This increased severity results from systemic injury of other organs or local pancreas-associated complications, or both; these injuries and complications result in increased mortality rates in patients with obesity and AP $[25,26]$.

Age and obesity have been identified by many studies as easily accessible and negative predictive factors in patients with AP [27-30]. An increased risk of early shock, pulmonary and kidney failure, and an extended hospital stay are associated with obesity [16, 19-24, 31]. De Waele et al. [32] found that in patients with AP, obesity is correlated with disease severity and an increased risk of local complications and mortality. Hong et al. [33] found that in addition to contributing to an increased risk of development of AP, obesity increases the risk of a poor prognosis in patients with AP. Sadr-Azodi et al. [20] study found that compared with individuals with a waist circumference between 75.1 and $85.0 \mathrm{~cm}$, those with a waist circumference $>105 \mathrm{~cm}$ had a statistically significant twofold increase in the overall risk of AP.

The body mass index (BMI) is a measure of obesity which is a BMI greater than or equal to $30 \mathrm{~kg} / \mathrm{m}^{2}$ [34]. Some results of clinical studies and studies using animal models suggest that BMI is associated with an increased risk of more severe AP [35]. Researchers who examined a Swedish cohort of 179 $\mathrm{AP}$ cases found a nonsignificant positive trend between BMI and AP risk [36]. A study of a US cohort of elderly women with acute or chronic pancreatitis (660 cases) revealed a positive association between BMI and AP [37]. Using a multivariate analysis, $\mathrm{Li}$ et al. found that a higher $\mathrm{BMI}$ is independently associated with increased risks of acute kidney injury, acute respiratory distress syndrome, and deep venous thrombosis [38]. A meta-analysis (739 cases) performed by Martinez et al. [39] revealed that obesity (BMI $\geq 30 \mathrm{~kg} / \mathrm{m}^{2}$ ) is a statistically significant risk factor for AP severity.
Emerging evidence suggests that an obesity paradox is present in patients with AP. Compared with patients with no obesity, patients with obesity and predicted severe AP, and no central adiposity, may experience better outcomes [40]. Therefore, obesity is not always an independent predictor for mortality and organ failure in patients with AP. Whether including obesity into scoring systems results in better prediction of severity remains to be determined.

The mechanisms via which obesity increases AP severity are unclear. One hypothesis assumes that chronic inflammation is associated with the adipose tissue, which releases pro-inflammatory cytokines. As a result, individuals with obesity are at a greater risk of lifestyle-related respiratory problems and chronic diseases $[35,41]$. A second hypothesis is that the pancreas-associated low-level inflammation in patients with obesity increases and becomes an even greater inflammatory reaction when AP does occur. Inflammation of intra and peri-pancreatic fat tissue is an important part of AP pathophysiology. Because patients with obesity have greater amounts of pancreatic fat, the resulting inflammation is more severe.

A third mechanism that has been proposed is related to the FL that occurs in patients with obesity. FL negatively affects liver-associated detoxification of inflammatory mediators during AP. In a fourth possible mechanism, obesity is suggested to be associated with an increased risk of extra-pancreatic complications (e.g., kidney failure, shock, respiratory insufficiency, and a fatal outcome) [42, 43]. A fifth proposed mechanism is that obesity may be associated with an intensification of the immune response and subsequently more severe pancreatic injury. A severity scoring system (APACH-O) was proposed by Papachristou et al. [44], which includes obesity as an independent predictor of AP outcome. These researchers proposed that obesity is associated with an increase in AP severity via amplification of the immune response to injury. Finally, the intra abdominal pressure is typically increased in patients with obesity. This pressure increase results in a ventilation/perfusion mismatch of the lungs because the diaphragm is elevated. When a systemic inflammatory state such as AP is present, the effect will be even more severe and might result in reduced oxygenation of, and enhancement of an existing injury to, the pancreas [31].

2.2. Hyperlipidemia. After gallstones (up to 60\%) [3] and alcohol (30\%) [3-5], hyperlipidemia (including chylomicronemia) is the third most common cause of AP; hyperlipidemia accounts for $4-10 \%$ of cases of AP worldwide [45]. However, study results indicate that in China, hypertriglyceridemia is the second major cause of AP [46, 47]. The values for incidence were as much as $12.3 \%$ in 2003 [48], 18.1\% in 2007 [49], and $25.6 \%$ in 2013 [46]. These values are much higher, compared with those in western countries. Severe hypertriglyceridemia accounts for $1-7 \%$ of all AP cases. In patients with primary or secondary hyperlipidemia, high circulating concentrations of triglycerides are associated with an increased risk of AP [45].

High triglyceride concentrations have been found in cases of AP with different etiologies. Therefore, hyperlipidemia is likely a primary precipitant of AP onset and an epiphenomenon $[50,51]$. The adjusted hazard ratio for the association between 
TABLE 1: Clinical studies on the relationships between different metabolic factors and occurrence, severity, and outcome of acute pancreatitis.

\begin{tabular}{|c|c|c|c|}
\hline Reference & Study design & Metabolic factors & Study conclusion \\
\hline De Waele et al. [32] & Prospective study & Obesity & $\begin{array}{l}\text { Body overweight and obesity represent a risk of more "severe" } \\
\text { disease and the number and type of complications increase in } \\
\text { categories of increasing BMI in acute biliary pancreatitis }\end{array}$ \\
\hline Hong et al. [33] & Meta-analysis & Obesity & $\begin{array}{c}\text { Obesity is not only associated with an increased risk of AP devel- } \\
\text { opment, but it is also a poor prognostic factor for AP }\end{array}$ \\
\hline Anderson et al. [56] & $\begin{array}{l}\text { Retrospective observational } \\
\text { study }\end{array}$ & Hyperlipdemia & $\begin{array}{l}\text { A reduction of serum triglyceride concentrations to } \\
<5.65 \mathrm{mmol} / \mathrm{L} \text { reduces abdominal pain in patients with AP and } \\
\text { improves clinical outcomes }\end{array}$ \\
\hline $\mathrm{Xu}$ et al. [68] & Retrospective cohort study & Fatty liver & $\begin{array}{l}\text { Fatty liver could influence the severity and clinical outcome and } \\
\text { may play a prognostic role in AP }\end{array}$ \\
\hline Mikolasevic et al. [71] & Retrospective cohort study & Fatty liver & $\begin{array}{l}\text { Presence of nonalcoholic fatty liver at admission can indicate a } \\
\text { higher risk of developing more severe forms of acute pancreatitis } \\
\text { and could be used as an additional prognostic tool }\end{array}$ \\
\hline Nawaz et al. [88] & Retrospective cohort study & Diabetes mellitus & Diabetes did not have an effect on the course of AP \\
\hline Shen et al. [87] & Retrospective cohort study & Diabetes mellitus & $\begin{array}{l}\text { Diabetes may adversely affect the disease process of AP, it seems } \\
\text { to protect patients from AP-related mortality }\end{array}$ \\
\hline
\end{tabular}

$\mathrm{BMI}=$ Body mass index, $\mathrm{AP}=$ Acute pancreatitis .

serum triglyceride concentration and the overall risk of AP is 1.21 per $1 \mathrm{mmol} / \mathrm{L}$ triglyceride increment [52]. Kota et al. found that a diet that includes omega-3 fatty acids and the use of fibrates are the most effective treatments for patients with hyperlipidemia and AP [53]. Severe hypertriglyceridemia can also be treated using insulin and dextrose infusion, which can activate or cause the release of the lipoprotein lipase enzyme $[54,55]$. The results of Anderson et al. [56] and Charlesworth et al. [51] studies indicated that a reduction of serum triglyceride concentrations to $<5.65 \mathrm{mmol} / \mathrm{L}$ reduces abdominal pain in patients with AP and improves clinical outcomes.

Only a few studies have investigated the effects of hypertriglyceridemia on acute biliary pancreatitis outcomes. The presence of hypertriglyceridemia is associated with a poor prognosis $[57,58]$. Zeng et al. study results suggested that high triglyceride concentrations are a risk factor for the development of severe AP and systemic and local complications in patients with AP [59].

No single mechanistic process has been found for the underlying pathogenesis of hypertriglyceridemic pancreatitis. Approximately one-fifth (15-20\%) of patients with severe hypertriglyceridemia will develop AP $[54,60]$. However, the etiology that initiates the disease process remains to be determined. Havel's theory [61] is the most commonly accepted pathogenic model. In this model, chylomicrons occlude pancreatic capillaries. The binding ability of albumin to aggregate into micelles with detergent properties is then overwhelmed by the hydrolysis of these triglyceride-rich lipoproteins, which releases high local concentrations of free fatty acids. The pancreatic vascular endothelium and acinar cells are subsequently injured by the free fatty acid micelle complexes. An acidic environment is created by the resulting ischemia, which induces increased free fatty acid toxicity. Cyclical micelle-free fatty acid production may partly contribute to the disease severity in patients with hyperlipidemic pancreatitis. Release and activation of pancreatic proteases and lipase and further autodigestion-associated injury are additional consequences of the acidic environment [57]. Pancreatitis would be induced in all cases with hyperchylomicronemia if Havel's proposed model was the only mechanism.

The hyperviscosity theory proposes that the presence of lipemia (i.e., high numbers of chylomicrons in the blood) contributes to the onset of hyperlipidemic pancreatitis [57]. Capillary blood flow in the pancreas is reduced by accumulation of chylomicrons in the microcirculation; ischemia then results. How hyperviscous blood induces selective pancreatic acinar cell ischemia remains unexplained.

Chang et al.s [62] genetic theory identifies cystic fibrosis transmembrane conductance regulator mutation/variant/haplotype and a tumor necrosis factor promoter polymorphism as independent risk factors. Compared with patients without pancreatitis, a higher proportion of patients with hypertriglyceridemia and AP have this genetic polymorphism [62]. The e-4 allele of the Apolipoprotein $E$ gene is more frequent in populations of patients with hyperlipidemic pancreatitis [63, 64]. Mitochondrial stress-induced direct tissue injury and lipotoxicity $[62,65,66]$ can cause cytokine and inflammatory cascade up-regulation and increase the risk that the patient will have a systemic inflammatory response [65]. Hyperlipidemic pancreatitis is a complex disorder with an underlying mechanism that is likely affected by genetic, metabolic, environmental, and patient-specific factors.

\section{Effects of Fatty Liver}

The incidence of FL as a metabolic disease is increasing annually, and it is an increasing public health problem worldwide. The prevalence of FL is approximately $30 \%$ in developed countries and nearly $10 \%$ in developing countries. It is an important reason for an abnormal liver function test result [67]. Associations between FL and AP have been investigated $[68,69]$. Patients with AP often also have FL because both diseases have the same contributing factors (e.g., hyperlipidemia, DM type 2, and obesity) [68-70]. 
$\mathrm{Xu}$ et al. assigned 2,671 patients with pancreatitis to an FL group or a nonFL group. Their study found that compared with nonFL group patients, FL group patients had a higher risk of death, and higher risks of severe AP and necrotizing AP. The values for incidence of local and systemic complications were also greater in the FL group. These results indicated that FL can affect severity and clinical outcome and may have prognostic value in patients with AP [68]. Mikolasevic et al. performed a retrospective analysis of a population of 822 patients hospitalized with AP. They found that the occurrence of moderately severe and severe AP and nonalcoholic FL disease (NAFLD) (odds ratio (OR) 2.13, 95\% CI 1.236-3.689) were independently associated. Compared with a nonNAFLD group of patients, patients with NAFLD have a higher, but not statistically significant, death rate $(5.6 \%$ vs. $4.3 \%$; $p=\mathrm{NS})$ [71]. Wu et al. examined the effect of NAFLD on AP severity. They found that compared with a nonNAFLD group, NAFLD has a clinically relevant effect on AP severity; the results suggested that the presence of NAFLD may be an early predictive factor for outcome in patients with AP [1]. Yoon et al. found that compared with patients with AP and without FL, patients with FL and AP had characteristics of more severe clinical disease (e.g., higher rates of local complications, persistent organ failure, and mortality). Even after adjusting for confounding variables (e.g., age, BMI, cause of AP) FL is a statistically significant risk factor for a severe disease course [70]. The results of a meta-analysis performed by Hou et al. to investigate the prognostic effect of FL on AP prognosis suggested that FL is independently associated with a severe clinical course of disease [72]. They also found a higher mortality in the FL-related AP group compared with the nonFL related AP group and a greater risk of systemic inflammatory response syndrome and local complications.

How FL increases the severity of pancreatitis remains to be determined. Populations of patients with FL often have greater rates of obesity [1] and hyperlipidemia. The pathogenesis via which obesity and hyperlipidemia lead to AP has been discussed above.

The inflammatory factor response easily results from the chronic inflammatory process that occurs in patients with obesity. As an inflammatory disease, FL also promotes chronic systemic inflammation [73-75] and likely exacerbates the severity of AP. The serum C-reactive protein level elevation that occurs in patients with FL suggests this chronic inflammatory process occurs $[70,76,77]$, and a chronic pro-inflammatory condition in patients with FL may increase the severity of the course of AP [72].

A decrease in serum Alpha-1-antitrypsin levels may lead to the excessive activation of inflammation. Alpha-1antitrypsin has significant anti-inflammatory properties because it affects a wide range of inflammatory cells (e.g., neutrophils, monocytes, macrophages, and mast cells). Results from rat and human AP models indicate that hepatic steatosis depresses Alpha-1-antitrypsin levels [78].

Kupffer cells are macrophages that reside in the liver. Approximately $70 \%$ of the liver's total macrophage numbers are Kupffer cells. They have an important role in AP pathogenesis via release of many different inflammatory factors [76].
During FL, Kupffer cells release of inflammatory factors is greatly increased. FL is also frequently accompanied by hyperlipidemia, which can induce accumulation of free radicals, disruption of the microcirculation, oxidative stress, and acinar necrosis in patients with AP [79-81].

The results of a recent study suggested that the PPAR $\alpha$ signaling and fatty acid degradation pathways are involved in the pathological processes that occur in patients with AP and FL (APFL). This result suggests that FL can increase the severity of pancreatitis via these pathways [82]. NAFLD patients often have disorders that increase Adipokine (e.g., $C P R, I L-6$, leptin) levels and reduce Adiponectin levels. These changes increase the risk of systemic inflammatory response syndrome [77].

\section{Effects of Diabetes Mellitus}

Some studies have examined the effect of pre-existing DM on AP outcome [42, 83-89]. DM may affect the course of AP [87-89]. Pang et al. found that the risk of AP is approximately $30 \%$ greater in patients with diabetes. This finding is consistent with findings of five record linkage studies that included a total of 6,524 cases of AP [61, 90-96]. Using a large prospective cohort study, Nogaard et al. [89] found that DM is a statistically significant factor associated with mortality in patients with AP $(P=0.016)$. A large retrospective cohort study of nearly 57,000 patients with AP revealed that patients with DM have a higher risk of severe AP compared with matched counterparts with no DM (OR 1.21, 95\% CI 1.16-1.26), a 58\% higher risk of ICU admission, and a 30\% greater risk of local complications [87]. However, the same study also found that despite this increased risk of severe AP, patients with DM and AP have a lower risk of mortality compared with those without DM (OR 0.77, 95\% CI 0.65-0.91). This finding is similar to that of another retrospective cohort study, which found that although length of stay is significantly longer for patients with pre-existing DM than those without DM (median 5 vs. $4 \mathrm{~d}$, $P<0.05)$, the results of a multivariate analysis indicated that severity of AP or in-hospital mortality is not significantly different between those with and without DM [88].

The details of the mechanisms via which DM affects outcomes in patients with AP have not been determined. It has been proposed that because patients with diabetes are likely to have a high comorbid burden (some of which is associated with AP etiologies such as hypertriglyceridemia and obesity), they will therefore have poorer reserves for a response to acute illness and an increased risk of severity and mortality $[87,88]$.

In summary, the studies that have investigated the effects of DM on outcomes in patients with AP have provided conflicting evidence. This is in part due to the study designs (retrospective cohort) used, which prevents the ability to isolate the effects of diabetes alone and control for confounding variables. Because the results from clinical studies are varied, more research is needed to determine the actual effects of DM on outcomes in patients with AP. 


\section{Conclusion}

The findings from this review indicate that FL is associated with poorer outcomes in patients with AP, including an increased risk of severe AP, an elevated systemic inflammatory response, and an increased mortality risk. The distribution and degree of obesity is also important to consider when predicting the effect of obesity on outcomes in patients with AP. DM and metabolic syndrome and, especially, obesity and hyperlipidemia, also adversely affect the course of AP. More research is required, which should include isolation of the effects of other metabolic factors and controlling for the effects of confounding variables.

Evidence of the mechanisms underlying these associations is limited, and more work is required to determine the detailed mechanisms via which obesity, DM, metabolic syndrome, and other comorbidities are related and affect outcomes in patients with AP. This review identified possible mechanisms by which obesity and other metabolic comorbidities affect the inflammatory response during AP. Further investigation into the roles of Adipokines and Cytokines is warranted. Patients with one or more comorbidities will likely require acute care at some point. The presence of comorbidities will increase the complexity of care. Therefore, understanding how chronic comorbidities affect the course and outcomes of disease is extremely important due to the increasing prevalence of these comorbidities worldwide. Use of large-scale, well-designed studies to determine the effects of chronic comorbidities on clinically meaningful outcomes should be a research priority.

\section{Data Availability}

The data used to support the findings of this study were supplied by Pujun Gao under license and so cannot be made freely available. Requests for access to these data should be made to Pujun Gao (gpj0411@163.com).

\section{Conflicts of Interest}

The authors declare that they have no conflicts of interest.

\section{Funding}

This study was supported by the Science and Technology Development Program of Jilin Province under grant no. 20190103079JH and the Youth Development Foundation of the First Hospital of Jilin University under grant no. JDYY102019004.

\section{References}

[1] D. Wu, M. Zhang, S. Xu et al., "Nonalcoholic fatty liver disease aggravated the severity of acute pancreatitis in patients," BioMed Research International, vol. 2019, p. 9583790, 2019.

[2] N. Crowther, M. Kahvo, and P. Chana, "Improving the management of acute pancreatitis in a district general hospital," BMJ Quality Improvement Reports, vol. 4, no. 1, p. p. u206577.w2642, 2015.
[3] C. E. Forsmark and J. Baillie, "AGA Institute technical review on acute pancreatitis," Gastroenterology, vol. 132, no. 5, pp. 2022-2044, 2007.

[4] Y. Lin, A. Tamakoshi, T. Hayakawa, M. Ogawa, and Y. Ohno, "Associations of alcohol drinking and nutrient intake with chronic pancreatitis: findings from a case-control study in Japan," The American Journal of Gastroenterology, vol. 96, no. 9, pp. 2622-2627, 2001.

[5] UK Working Party on Acute Pancreatitis, "UK guidelines for the management of acute pancreatitis," Gut, vol. 54, no. suppl_3, pp. iiil-iii9, 2005.

[6] D. Stimac, I. Mikolasevic, I. Krznaric-Zrnic, M. Radic, and S. Milic, "Epidemiology of acute pancreatitis in the north adriatic region of Croatia during the last ten years," Gastroenterology Research and Practice, vol. 2013, Article ID 956149, pp. 1-5, 2013.

[7] WHO, "Guidelines for the screening, care and treatment of persons with chronic hepatitis $\mathrm{C}$ infection. Updated version April 2016," https://www.who.int/hepatitis/publications/ hepatitis-c-guidelines-2016/en/

[8] G. Danaei, M. M. Finucane, Y. Lu et al., "National regional, and global trends in fasting plasma glucose and diabetes prevalence since 1980: systematic analysis of health examination surveys and epidemiological studies with 370 country-years and 2.7 million participants," The Lancet (London, England), vol. 378, no. 9785, pp. 31-40, 2011.

[9] K. G. M. M. Alberti, P. Zimmet, and J. Shaw, "Metabolic syndrome-a new world-wide definition. A consensus statement from the international diabetes federation," Diabetic Medicine: A Journal of the British Diabetic Association, vol. 23, no. 5, pp. 469-480, 2006.

[10] R. L. Goodger, V. M. Asrani, J. A. Windsor, and M. S. Petrov, "Impact of metabolic comorbidities on outcomes of patients with acute pancreatitis: a scoping review," Panminerva Medica, vol. 58, no. 1, pp. 86-93, 2016.

[11] J. E. Shaw, R. A. Sicree, and P. Z. Zimmet, "Global estimates of the prevalence of diabetes for 2010 and 2030," Diabetes Research and Clinical Practice, vol. 87, no. 1, pp. 4-14, 2010.

[12] S. L. Das, P. P. Singh, A. R. Phillips, R. Murphy, J. A. Windsor, and M. S. Petrov, "Newly diagnosed diabetes mellitus after acute pancreatitis: a systematic review and meta-analysis," Gut, vol. 63, no. 5, pp. 818-831, 2014.

[13] G. M. Reaven, "Banting lecture 1988. Role of insulin resistance in human disease," Diabetes, vol. 37, no. 12, pp. 1595-1607, 1988.

[14] I.-C. Hwang, K.-K. Kim, S.-H. Jee, and H.-C. Kang, "A comparison of predictability of cardiovascular events between each metabolic component in patients with metabolic syndrome based on the revised National Cholesterol Education Program criteria," Yonsei medical journal, vol. 52, no. 2, pp. 220-226, 2011.

[15] M. Larvin and M. J. Mcmahon, "APACHE-II score for assessment and monitoring of acute pancreatitis," Lancet (London, England), vol. 334, no. 8656, pp. 201-205, 1989.

[16] S. Sawalhi, H. Al-Maramhy, A. I. Abdelrahman, S. E. G. Allah, and S. Al-Jubori, "Does the presence of obesity and/or metabolic syndrome affect the course of acute pancreatitis?," A prospective study. Pancreas, vol. 43, no. 4, pp. 565-570, 2014.

[17] L.-H. Deng, P. Xue, Q. Xia, X.-N. Yang, and M.-H. Wan, "Effect of admission hypertriglyceridemia on the episodes of severe acute pancreatitis," World Journal of Gastroenterology, vol. 14, no. 28 , pp. $4558-4561,2008$. 
[18] I. Mikolasevic, S. Milic, L. Orlic et al., "Metabolic syndrome and acute pancreatitis," European Journal of Internal Medicine, vol. 32, pp. 79-83, 2016.

[19] S. Wang, S. Li, Q. Feng, X. Feng, L. Xu, and Q. Zhao, "Overweight is an additional prognostic factor in acute pancreatitis: a metaanalysis," Pancreatology: Official Journal of the International Association of Pancreatology (IAP)... [et al], vol. 11, no. 2, pp. 92-98, 2011.

[20] O. Sadr-Azodi, N. Orsini, A. Andrén-Sandberg, and A. Wolk, "Abdominal and total adiposity and the risk of acute pancreatitis: a population-based prospective cohort study," American Journal of Gastroenterology, vol. 108, no. 1, pp. 133-139, 2013.

[21] J. Katuchova, J. Bober, P. Harbulak et al., "Obesity as a risk factor for severe acute pancreatitis patients," Wiener klinische Wochenschrift, vol. 126, no. 7-8, pp. 223-227, 2014.

[22] R. C. Turner and R. McDermott, "Clinical predictors of severe acute pancreatitis: value-adding the view from the end of the bed," ANZ Journal of Surgery, vol. 84, no. 9, pp. 672-676, 2014.

[23] S. G. Krishna, A. Hinton, V. Oza et al., "Morbid obesity is associated with adverse clinical outcomes in acute pancreatitis: a propensity-matched study," The American journal of gastroenterology, vol. 110, no. 11, pp. 1608-1619, 2015.

[24] A. Murata, M. Ohtani, K. Muramatsu, S. Kobori, S. Tomioka, and S. Matsuda, "Impact of obesity on outcomes of paediatric acute pancreatitis based on a national administrative database," Pediatric Obesity, vol. 11, no. 3, pp. 174-180, 2016.

[25] J. Martinez, J. Sánchez-Payá, J. M. Palazón, J. R. Aparicio, A. Picó, and M. Pérez-Mateo, "Obesity: a prognostic factor of severity in acute pancreatitis," Pancreas, vol. 19, no. 1, pp. 15-20, 1999.

[26] C. D. Johnson, S. K. C. Toh, and M. J. Campbell, "Combination of APACHE-II score and an obesity score (APACHE-O) for the prediction of severe acute pancreatitis," Pancreatology: Official Journal of the International Association of Pancreatology (IAP)... [et al], vol. 4, no. 1, pp. 1-6, 2004.

[27] C. S. Pitchumoni, N. Patel, and M, Shah P., "Factors influencing mortality in acute pancreatitis: can we alter them?", Journal of Clinical Gastroenterology, vol. 39, no. 9, pp. 798-814, 2005.

[28] A. B. Lowenfels, P. Maisonneuve, and T. Sullivan, "The changing character of acute pancreatitis: epidemiology, etiology, and prognosis," Current Gastroenterology Reports, vol. 11, no. 2, pp. 97-103, 2009.

[29] L. Bonfrate, D. Q.-H. Wang, G. Garruti, and P. Portincasa, "Obesity and the risk and prognosis of gallstone disease and pancreatitis," Best Practice \& Research Clinical Gastroenterology, vol. 28, no. 4, pp. 623-635, 2014.

[30] C. Acharya, S. Navina, and V. P. Singh, "Role of pancreatic fat in the outcomes of pancreatitis," Pancreatology: Official Journal of the International Association of Pancreatology (IAP) [et al], vol. 14, no. 5, pp. 403-408, 2014.

[31] J.-L. Frossard, P. Lescuyer, and C. M. Pastor, "Experimental evidence of obesity as a risk factor for severe acute pancreatitis," World Journal of Gastroenterology, vol. 15, no. 42, pp. 5260-5265, 2009.

[32] B. De Waele, B. Vanmierlo, Y. Van Nieuwenhove, and G. Delvaux, "Impact of body overweight and class I, II and III obesity on the outcome of acute biliary pancreatitis," Pancreas, vol. 32, no. 4, pp. 343-345, 2006.

[33] S. Hong, B. Qiwen, J. Ying, A. Wei, and T. Chaoyang, "Body mass index and the risk and prognosis of acute pancreatitis: a metaanalysis," European Journal of Gastroenterology \& Hepatology, vol. 23, no. 12, pp. 1136-1143, 2011.
[34] WHO, "Obesity and overweight 2019," https://www.who.int/ news-room/fact-sheets/detail/obesity-and-overweight.

[35] T. Ikeura, K. Kato, M. Takaoka et al., "A body mass index $</=25$ $\mathrm{kg} / \mathrm{m}(2)$ is associated with a poor prognosis in patients with acute pancreatitis: a study of Japanese patients," Hepatobiliary \& Pancreatic Diseases International: HBPD INT, vol. 16, no. 6, pp. 645-651, 2017.

[36] B. Lindkvist, S. Appelros, J. Manjer, G. Berglund, and A. Borgstrom, "A prospective cohort study of smoking in acute pancreatitis," Pancreatology: Official Journal of the International Association of Pancreatology (IAP)...[et al], vol. 8, no. 1, pp. 63-70, 2008.

[37] A. E. Prizment, E. H. Jensen, A. M. Hopper, B. A. Virnig, and K. E. Anderson, "Risk factors for pancreatitis in older women: the iowa Women's Health Study," Annals of Epidemiology, vol. 25, no. 7, pp. 544-548, 2015.

[38] X. Li, L. Ke, J. Dong et al., "Significantly different clinical features between hypertriglyceridemia and biliary acute pancreatitis: a retrospective study of 730 patients from a tertiary center," $B M C$ Gastroenterology, vol. 18, no. 1, p. 89, 2018.

[39] J. Martinez, C. D. Johnson, J. Sanchez-Paya, E. de Madaria, G. Robles-Diaz, and M. Perez-Mateo, "Obesity is a definitive risk factor of severity and mortality in acute pancreatitis: an updated meta-analysis," Pancreatology: Official Journal of the International Association of Pancreatology (IAP) [et al], vol. 6, no. 3, pp. 206-209, 2006.

[40] R. Premkumar, A. R. J. Phillips, M. S. Petrov, and J. A. Windsor, "The clinical relevance of obesity in acute pancreatitis: targeted systematic reviews," Pancreatology: Official Journal of the International Association of Pancreatology (IAP) [et al], vol. 15, no. 1, pp. 25-33, 2015.

[41] L.-G. Hersoug, P. Møller, and S. Loft, "Gut microbiota-derived lipopolysaccharide uptake and trafficking to adipose tissue: implications for inflammation and obesity," Obesity Reviews: An Official Journal of the International Association for the Study of Obesity, vol. 17, no. 4, pp. 297-312, 2016.

[42] P. G. Lankisch and C. A. Schirren, "Increased body weight as a prognostic parameter for complications in the course of acute pancreatitis," Pancreas, vol. 5, no. 5, pp. 626-629, 1990.

[43] K. B. Blomgren, A. Sundstrom, G. Steineck, and B. E. Wiholm, "Obesity and treatment of diabetes with glyburide may both be risk factors for acute pancreatitis," Diabetes Care, vol. 25, no. 2, pp. 298-302, 2002.

[44] G. I. Papachristou, D. J. Papachristou, H. Avula, A. Slivka, and D. C. Whitcomb, "Obesity increases the severity of acute pancreatitis: performance of APACHE-O score and correlation with the inflammatory response," Pancreatology: Official Journal of the International Association of Pancreatology (IAP) [et al], vol. 6, no. 4, pp. 279-285, 2006.

[45] A. Adiamah, E. Psaltis, M. Crook, and D. N. Lobo, "A systematic review of the epidemiology, pathophysiology and current management of hyperlipidaemic pancreatitis," Clinical Nutrition (Edinburgh, Scotland), vol. 37, no. 6, pp. 1810-1822, 2018.

[46] G. Yin, X. Cang, G. Yu et al., "Different clinical presentations of hyperlipidemic acute pancreatitis: a retrospective study," Pancreas, vol. 44, no. 7, pp. 1105-1110, 2015.

[47] Y. Zhu, X. Pan, H. Zeng et al., "A study on the etiology, severity, and mortality of 3260 patients with acute pancreatitis according to the revised atlanta classification in jiangxi, China over an 8-year period," Pancreas, vol. 46, no. 4, pp. 504-509, 2017. 
[48] M. C. Chang, C. H. Su, M. S. Sun et al., "Etiology of acute pancreatitis-a multi-center study in Taiwan," Hepatogastroenterology, vol. 50, no. 53, pp. 1655-1667, 2003.

[49] J. M. Qian, "Reviewing the etiology, diagnosis and treatment of acute pancreatitis in China," Zhonghua Nei ke Za Zhi, vol. 46, no. 12, pp. 979-980, 2007.

[50] J. E. Domínguez-Muñoz, F. Jünemann, and P. Malfertheiner, "Hyperlipidemia in acute pancreatitis. Cause or epiphenomenon? International Journal of Pancreatology: Official Journal of the International Association of," Pancreatology., vol. 18, no. 2, pp. 101-106, 1995.

[51] A. Charlesworth, A. Steger, and M. A. Crook, "Acute pancreatitis associated with severe hypertriglyceridaemia; a retrospective cohort study," International journal of surgery (London, England), vol. 23, no. Pt A, pp. 23-27, 2015.

[52] B. Lindkvist, S. Appelros, S. Regner, and J. Manjer, "A prospective cohort study on risk of acute pancreatitis related to serum triglycerides, cholesterol and fasting glucose," Pancreatology: Official Journal of the International Association of Pancreatology (IAP) [et al], vol. 12, no. 4, pp. 317-324, 2012.

[53] S. Kota, S. Kota, S. Jammula, S. V. S. Krishna, and K. Modi, "Hypertriglyceridemia-induced recurrent acute pancreatitis: a case-based review," Indian Journal of Endocrinology and Metabolism, vol. 16, no. 1, pp. 141-143, 2012.

[54] S. Sandhu, A. Al-Sarraf, C. Taraboanta, J. Frohlich, and G. A. Francis, "Incidence of pancreatitis, secondary causes, and treatment of patients referred to a specialty lipid clinic with severe hypertriglyceridemia: a retrospective cohort study," Lipids in Health and Disease, vol. 10, no. 1, p. 157, 2011.

[55] L. Berglund, J. D. Brunzell, A. C. Goldberg et al., "Evaluation and treatment of hypertriglyceridemia: an endocrine society clinical practice guideline," The Journal of Clinical Endocrinology \& Metabolism, vol. 97, no. 9, pp. 2969-2989, 2012.

[56] F. Anderson, S. Z. Mbatha, and S. R. Thomson, "The early management of pancreatitis associated with hypertriglyceridaemia," South African Journal of Surgery, vol. 49, no. 2, pp. 82-84, 2011.

[57] P. Valdivielso, A. Ramirez-Bueno, and N. Ewald, "Current knowledge of hypertriglyceridemic pancreatitis," European Journal of Internal Medicine, vol. 25, no. 8, pp. 689-694, 2014.

[58] R. A. Carr, B. J. Rejowski, G. A. Cote, H. A. Pitt, and N. J. Zyromski, "Systematic review of hypertriglyceridemiainduced acute pancreatitis: a more virulent etiology?", Pancreatology: Official Journal of the International Association of Pancreatology (IAP) [et al], vol. 16, no. 4, pp. 469-476, 2016.

[59] Y. Zeng, W. Zhang, Y. Lu, C. Huang, and X. Wang, "Impact of hypertriglyceridemia on the outcome of acute biliary pancreatitis," The American Journal of the Medical Sciences, vol. 348, no. 5, pp. 399-402, 2014.

[60] J. Scherer, V. P. Singh, C. S. Pitchumoni, and D. Yadav, "Issues in hypertriglyceridemic pancreatitis: an update," Journal of Clinical Gastroenterology, vol. 48, no. 3, pp. 195-203, 2014.

[61] R. J. Havel, "Pathogenesis, differentiation and management of hypertriglyceridemia," Advances in Internal Medicine, vol. 15, pp. 117-154, 1969.

[62] Y.-T. Chang, M.-C. Chang, T.-C. Su et al., "Association of cystic fibrosis transmembrane conductance regulator (CFTR) mutation/variant/haplotype and tumor necrosis factor (TNF) promoter polymorphism in hyperlipidemic pancreatitis," Clinical Chemistry, vol. 54, no. 1, pp. 131-138, 2008.
[63] R. Ivanova, S. Puerta, A. Garrido et al., "Triglyceride levels and apolipoprotein E polymorphism in patients with acute pancreatitis," Hepatobiliary \& Pancreatic Diseases International (HBPD INT), vol. 11, no. 1, pp. 96-101, 2012.

[64] A. Charlesworth, A. Steger, and M. A. Crook, "Hyperlipidemic acute pancreatitis and the apolipoprotein E4 allele," Pancreas, vol. 46, no. 1, pp. e3-e4, 2017.

[65] Y.-T. Chang, M.-C. Chang, T.-C. Su et al., "Lipoprotein lipase mutation S447X associated with pancreatic calcification and steatorrhea in hyperlipidemic pancreatitis," Journal of Clinical Gastroenterology, vol. 43, no. 6, pp. 591-596, 2009.

[66] Y. Zeng, X. Wang, W. Zhang, K. Wu, and J. Ma, "Hypertriglyceridemia aggravates ER stress and pathogenesis of acute pancreatitis," Hepatogastroenterology, vol. 59, no. 119, pp. 2318-2326, 2012.

[67] G. Bedogni, V. Nobili, and C. Tiribelli, "Epidemiology of fatty liver: an update," World Journal of Gastroenterology, vol. 20, no. 27, pp. 9050-9054, 2014.

[68] C. Xu, Z. Qiao, Y. Lu et al., "Influence of fatty liver on the severity and clinical outcome in acute pancreatitis," PLOS ONE, vol. 10, no. 11, p. e0142278, 2015.

[69] X. Qi, Y. Hou, and X. Guo, "Severe fatty liver disease and acute pancreatitis: is there a correlation between them?", Clinical and Experimental Hepatology, vol. 4, pp. 127-130, 2016.

[70] S. B. Yoon, I. S. Lee, M. H. Choi et al., "Impact of fatty liver on acute pancreatitis severity," Gastroenterology Research and Practice, vol. 2017, Article ID 10.1155/2017/4532320, pp. 1-7, 2017.

[71] I. Mikolasevic, L. Orlic, G. Poropat et al., "Nonalcoholic fatty liver and the severity of acute pancreatitis," European Journal of Internal Medicine, vol. 38, pp. 73-78, 2017.

[72] S. Hou, X. Tang, H. Cui et al., "Fatty liver disease is associated with the severity of acute pancreatitis: a systematic review and meta-analysis," International Journal of Surgery (London, England), vol. 65, pp. 147-153, 2019.

[73] J. Wang, Z. Feng, Y. Li, Q. Li, and X. Tao, "Association of tumor necrosis factor-alpha gene promoter polymorphism at sites -308 and -238 with non-alcoholic fatty liver disease: a meta-analysis," Journal of gastroenterology and hepatology, vol. 27, no. 4, pp. 670-676, 2012.

[74] J. Luo, L. Xu, J. Li, and S. Zhao, "Nonalcoholic fatty liver disease as a potential risk factor of cardiovascular disease," European Journal of Gastroenterology \& Hepatology, vol. 27, no. 3, pp. 193-199, 2015.

[75] F. Zamani, B. Amirkalali, M. R. Sohrabi et al., "Erythrocyte membrane fatty acid profile \& serum cytokine levels in patients with non-alcoholic fatty liver disease," The Indian Journal of Medical Research, vol. 147, no. 4, pp. 352-360, 2018.

[76] G. Baffy, "Kupffer cells in non-alcoholic fatty liver disease: the emerging view," Journal of Hepatology, vol. 51, no. 1, pp. 212-223, 2009.

[77] C. E. Ndumele, K. Nasir, R. D. Conceicao, J. A. M. Carvalho, R. S. Blumenthal, and R. D. Santos, "Hepatic steatosis, obesity, and the metabolic syndrome are independently and additively associated with increased systemic inflammation," Arteriosclerosis, Thrombosis, and Vascular Biology, vol. 31, no. 8, pp. 1927-1932, 2011.

[78] Q. Wang, J. Du, P. Yu et al., "Hepatic steatosis depresses alpha-1antitrypsin levels in human and rat acute pancreatitis," Scientific Reports, vol. 5, no. 1, Article ID 17833, 2015. 
[79] J. Ditzel and E. H. Thaysen, "Increased hemoglobin-oxygen affinity in patients with pancreatitis associated with type I and V hyperlipoproteinemia," Advances in Experimental Medicine and Biology, vol. 94, pp. 423-428, 1977.

[80] H. Nagai, H. Henrich, P.-H. Wunsch, W. Fischbach, and J. Mössner, "Role of pancreatic enzymes and their substrates in autodigestion of the pancreas. In vitro studies with isolated rat pancreatic acini," Gastroenterology, vol. 96, no. 3, pp. 838-847, 1989.

[81] A. Van Gossum, P. Closset, E. Noel, M. Cremer, and J. Neve, "Deficiency in antioxidant factors in patients with alcoholrelated chronic pancreatitis," Digestive Diseases and Sciences, vol. 41, no. 6, pp. 1225-1231, 1996.

[82] Q. Wang, H. Yan, G. Wang et al., "RNA sequence analysis of rat acute experimental pancreatitis with and without fatty liver: a gene expression profiling comparative study," Scientific Reports, vol. 7, no. 1, p. 734, 2017.

[83] D. Yadav and A. B. Lowenfels, "The epidemiology of pancreatitis and pancreatic cancer," Gastroenterology, vol. 144, no. 6, pp. 1252-1261, 2013.

[84] A. Lonardo, S. Ballestri, G. Marchesini, P. Angulo, and P. Loria, "Nonalcoholic fatty liver disease: a precursor of the metabolic syndrome," Digestive and Liver Disease: Official Journal of the Italian Society of Gastroenterology and the Italian Association for the Study of the Liver, vol. 47, no. 3, pp. 181-190, 2015.

[85] S. Majumder and S. T. Chari, "Chronic pancreatitis," The Lancet (London, England), vol. 387, no. 10031, pp. 1957-1966, 2016.

[86] M. S. Petrov and J. A. Windsor, "Classification of the severity of acute pancreatitis: how many categories make sense?," The American Journal of Gastroenterology, vol. 105, no. 1, pp. 74-76, 2010.

[87] H.-N. Shen, C.-L. Lu, and C.-Y. Li, "Effect of diabetes on severity and hospital mortality in patients with acute pancreatitis: a national population-based study," Diabetes Care, vol. 35, no. 5, pp. 1061-1066, 2012.

[88] H. Nawaz, M. O'Connell, G. I. Papachristou, and D. Yadav, "Severity and natural history of acute pancreatitis in diabetic patients," Pancreatology: Official Journal of the International Association of Pancreatology (IAP) [et al], vol. 15, no. 3, pp. 247-252, 2015.

[89] C. Nøjgaard, "Prognosis of acute and chronic pancreatitis - a 30-year follow-up of a Danish cohort," Danish Medical Bulletin, vol. 57, no. 12, B4228 pages, 2010.

[90] R. A. Noel, D. K. Braun, R. E. Patterson, and G. L. Bloomgren, "Increased risk of acute pancreatitis and biliary disease observed in patients with type 2 diabetes: a retrospective cohort study," Diabetes Care, vol. 32, no. 5, pp. 834-838, 2009.

[91] A. Gonzalez-Perez, R. G. Schlienger, and L. A. G. Rodriguez, "Acute pancreatitis in association with type 2 diabetes and antidiabetic drugs: a population-based cohort study," Diabetes Care, vol. 33, no. 12, pp. 2580-2585, 2010.

[92] R. Garg, W. Chen, and M. Pendergrass, "Acute pancreatitis in type 2 diabetes treated with exenatide or sitagliptin: a retrospective observational pharmacy claims analysis," Diabetes Care, vol. 33, no. 11, pp. 2349-2354, 2010.

[93] C. J. Girman, T. D. Kou, B. Cai et al., "Patients with type 2 diabetes mellitus have higher risk for acute pancreatitis compared with those without diabetes," Diabetes, Obesity and Metabolism, vol. 12, no. 9, pp. 766-771, 2010.

[94] S.-W. Lai, C.-H. Muo, K.-F. Liao, F.-C. Sung, and P.-C. Chen, "Risk of acute pancreatitis in type 2 diabetes and risk reduction on anti-diabetic drugs: a population-based cohort study in Taiwan," The American Journal of Gastroenterology, vol. 106, no. 9, pp. 1697-1704, 2011.

[95] H. Urushihara, M. Taketsuna, Y. Liu et al., "Increased risk of acute pancreatitis in patients with type 2 diabetes: an observational study using a japanese hospital database," PLoS ONE, vol. 7, no. 12, p. e53224, 2012.

[96] Y. Pang, C. Kartsonaki, I. Turnbull et al., "Metabolic and lifestyle risk factors for acute pancreatitis in Chinese adults: a prospective cohort study of 0.5 million people," PLOS Medicine, vol. 15, no. 8, p. e1002618, 2018. 


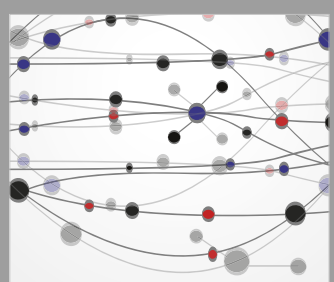

The Scientific World Journal
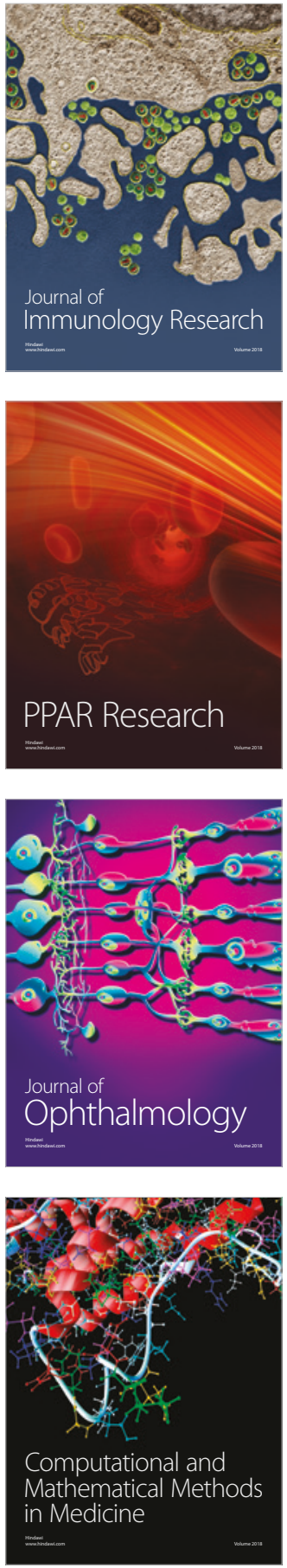

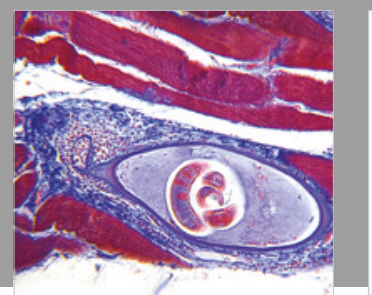

Gastroenterology Research and Practice

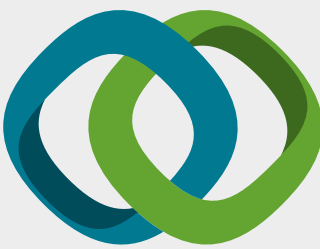

\section{Hindawi}

Submit your manuscripts at

www.hindawi.com
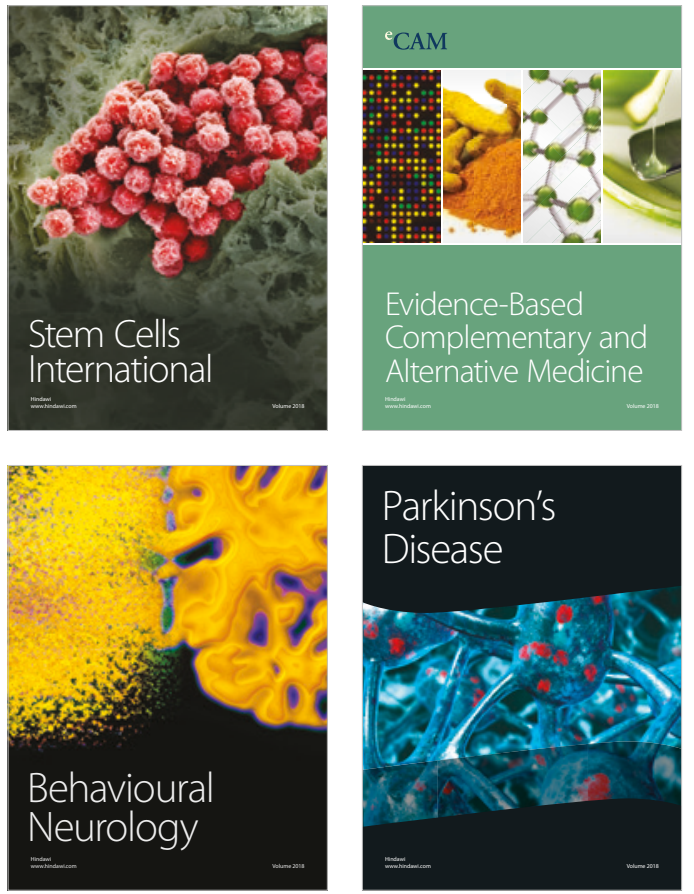

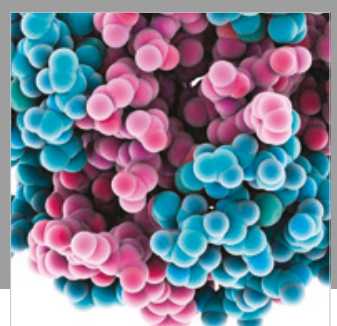

ournal of

Diabetes Research

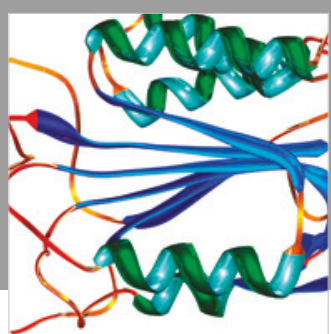

Disease Markers
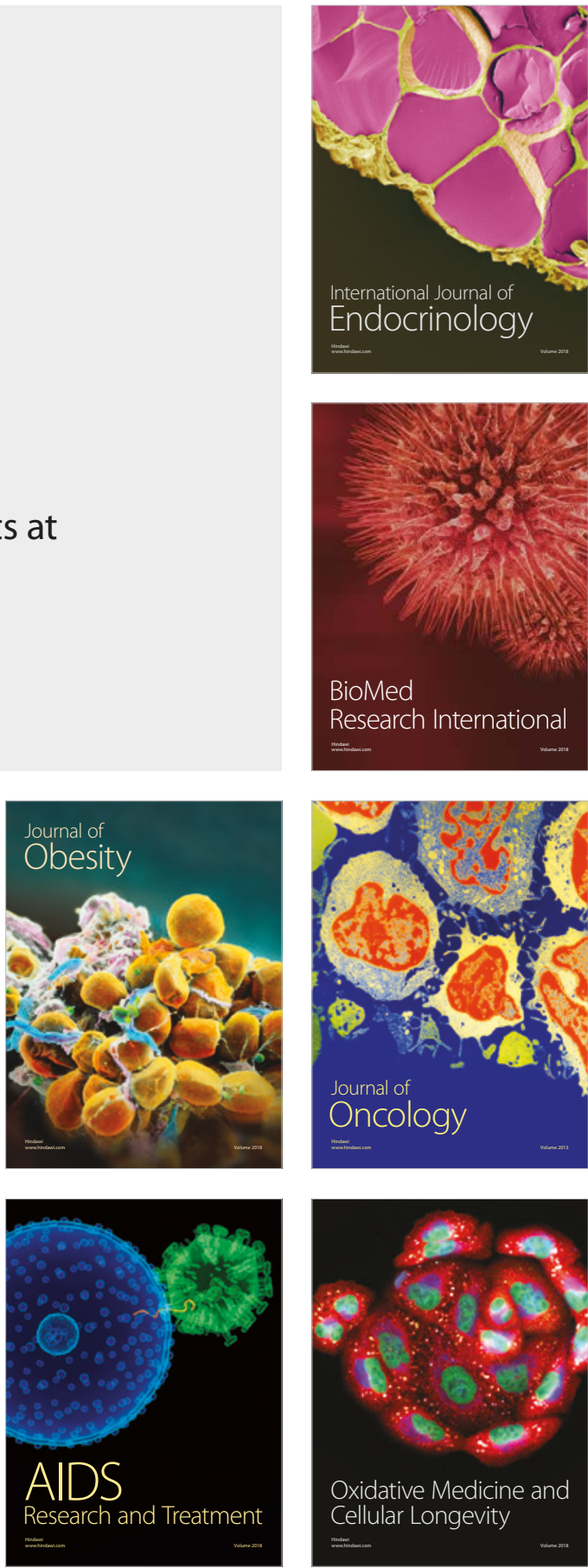